\title{
Patients' attitudes about the use of placebo treatments: telephone survey
}

\author{
(C) (1) $\Theta$ OPEN ACCESS
}

\author{
Sara Chandros Hull bioethicist ${ }^{12}$, Luana Colloca research fellow ${ }^{234}$, Andrew Avins research scientist ${ }^{5}$, \\ Nancy P Gordon research scientist ${ }^{5}$, Carol P Somkin research scientist ${ }^{5}$, Ted J Kaptchuk director ${ }^{6}$, \\ Franklin G Miller bioethicist ${ }^{23}$
}

${ }^{1}$ Office of the Clinical Director, National Human Genome Research Institute, National Institutes of Health, Bethesda, MD, USA; ${ }^{2}$ Department of Bioethics, Clinical Center, National Institutes of Health, Bethesda, MD, USA; ${ }^{3}$ National Institute of Mental Health, National Institutes of Health, Bethesda, MD, USA; ${ }^{4}$ National Center for Complementary and Alternative Medicine, National Institutes of Health, Bethesda, MD, USA; ${ }^{5}$ Division of

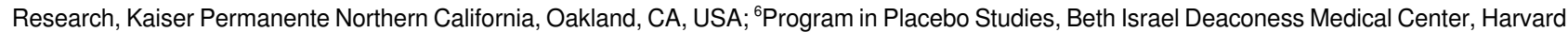
Medical School, Boston, MA, USA

\begin{abstract}
Objective To examine the attitudes of US patients about the use of placebo treatments in medical care.

Design One time telephone surveys.

Setting Northern California.

Participants 853 members of Kaiser Permanente Northern California, aged 18-75, who had been seen by a primary care provider for a chronic health problem at least once in the prior six months.

Results The response rate was 53.4\% (853/1598) of all members who were eligible to participate, and $73.2 \%(853 / 1165)$ of all who could be reached by telephone. Most respondents (50-84\%) judged it acceptable for doctors to recommend placebo treatments under conditions that varied according to doctors' level of certainty about the benefits and safety of the treatment, the purpose of the treatment, and the transparency with which the treatment was described to patients. Only $21.9 \%$ of respondents judged that it was never acceptable for doctors to recommend placebo treatments. Respondents valued honesty by physicians regarding the use of placebos and believed that non-transparent use could undermine the relationship between patients and physicians.
\end{abstract}

Conclusions Most patients in this survey seemed favorable to the idea of placebo treatments and valued honesty and transparency in this context, suggesting that physicians should consider engaging with patients to discuss their values and attitudes about the appropriateness of using treatments aimed at promoting placebo responses in the context of clinical decision making.

\section{Introduction}

Several recent surveys of physicians have documented their use of placebo treatments in clinical practice. ${ }^{1-4}$ In a recent US survey of internists and rheumatologists, half reported that they have prescribed placebo treatments, defined as treatments "whose benefits derive from positive patient expectations rather than from the physiologic or pharmacologic mechanism of the treatment itself." ${ }^{4}$ These placebo treatments included active agents (more often than inert substances) such as vitamins or analgesics that a physician prescribed to promote positive placebo effects rather than specific pharmacologic or physiologic effects. How these surveys described placebo treatments varied considerably, suggesting that there are conceptual nuances regarding the use of such treatments in clinical care that need to be better understood. ${ }^{5}$ Although some distinguish between pure and impure (or inert and active) placebos, it is the lack of specific efficacy that is based on the inherent properties of a compound that makes an intervention a placebo. ${ }^{6}$

The prescription of placebo treatments as part of medical care is ethically controversial. Their use has been criticized because the practice is thought to involve deception, thereby violating patient autonomy, ${ }^{78}$ because of concerns about the compatibility of placebo treatments with evidence based medicine, and because the risks introduced by some placebo treatments outweigh the possible benefits of their use, as in the case of prescribing antibiotics for viral infection. ${ }^{9}$ US clinical practice guidelines prohibit the use of placebo treatments without a patient's knowledge, citing concerns about undermining trust and compromising the patient-physician relationship. ${ }^{10}$ Despite these concerns, some have argued that use of placebo treatments can be justified when they are effective, at least in certain cases. ${ }^{11}{ }^{12}$ Furthermore, some placebo treatments may be effective without the use of deception. ${ }^{13}$ A recent randomized trial of open "inert" placebo pills in patients with irritable bowel syndrome, offered without deception but with the suggestion of benefit from the placebo response, found clinically significant 
improvement of symptoms compared with those receiving no treatment. ${ }^{14}$

The perspectives of US patients have been missing in the debate over the use of placebo treatments in clinical practice. Although one study explored how US participants in clinical trials conceptualize the placebo effect, ${ }^{15}$ little research has examined how patients think about placebo treatments. Surveys in other countries have described patients' attitudes toward use of placebo treatments. ${ }^{36-18}$ These studies suggest that patients are open to the use of placebo treatments in specific circumstances, sometimes even more so than clinicians, although they wish to be informed about the receipt of such therapies. However, the generalizability of the findings needs to be interpreted cautiously, given cultural differences and the various definitions of "placebo treatment" used in the surveys.

Data on patients' perspectives are essential to understanding this issue and formulating rational clinical policies. For example, if patients are generally accepting of their physicians' use of placebos, then the ethical perspectives on placebo use may need to be reconsidered. If patients are generally opposed to placebo use, then many physicians may need more education about their behavior with respect to their clinical use of placebos. And if there exists large variation in patients' viewpoints, physicians will need to learn how to elicit their patients' perspectives to act in concert with their desires; in this case, blanket prohibition of clinical placebo use may be misguided.

To probe the attitudes of US patients regarding placebo treatments, we conducted a survey of adult members of a large Northern California health plan. Our survey utilized a carefully constructed definition of "placebo treatments," used a combination of general questions and detailed scenarios, and included a large and demographically diverse sample of patients.

\section{Methods}

The sample for this survey was adult members of Kaiser Permanente Northern California aged 18-75 who had been seen in an outpatient clinic for a chronic health problem at least once in the prior six months. The demographic and health characteristics of the Kaiser Permanente Northern California adult population are similar to the population of insured adults in Northern California, with the exception of a smaller percentage of adults at low levels of educational attainment. ${ }^{19}$ After assigning all eligible members a random study identifier, we randomly selected an age stratified sample (10 year age groups), with members contacted to participate in the survey in consecutive numerical order from the randomly generated list. An introductory letter described the study goals and offered members an opportunity to opt out of the study. We telephoned those who did not opt out and asked them to complete the survey by phone. We excluded people who had dementia or could not participate in a telephone interview because of communication barriers (hearing, language). Potential participants were telephoned up to five times before being dropped from the survey pool. Respondents were sent a $\$ 20(£ 13 ; € 15)$ gift card after participating in the interview. Participants gave informed consent before taking part in the survey.

The survey was developed through a process that included patient focus groups in Baltimore, Maryland, expert review, and pretesting of the telephone interviews with 20 members of Kaiser Permanente Northern California. Trained interviewers administered the survey using a computer aided telephone interview system, which took respondents an average of 20 minutes to complete. Respondents were initially asked about their beliefs regarding the connection between the mind, body, and illness, and their knowledge of placebos and the placebo effect. They were then provided with an operational definition of "placebo treatment": "A patient experiences a placebo effect when they get better after taking a treatment, not because of the treatment itself, but because the patient expects they will benefit from the treatment. For the rest of this survey, we will ask you to assume that a placebo treatment is a treatment that only works because it can produce a placebo effect. Placebo treatments can be sugar pills or other treatments used to create a placebo effect." This definition was based on previous surveys of physicians and our experience with patient focus groups.

Following this explanation, we asked respondents about their beliefs regarding placebo mechanisms and the acceptability of doctors recommending placebo treatments, using both general questions and scenarios that varied the nature of the placebo treatment, the treatment indication, and the manner in which the physician described the treatment to the patient. Respondents were randomized to two different versions of scenario 1 (box), which varied according to whether or not the patient was aware that the doctor was recommending a placebo treatment.

Respondents were also asked about their willingness to take placebos in different scenarios.

\section{Statistical analysis}

We analyzed data using SAS version 9.1. For most variables, responses were collapsed into positive (definitely or probably yes, strongly or somewhat agree) versus negative (probably or definitely not, somewhat or strongly disagree), or one extreme (for example, definitely yes) versus other. To test for significance we used the TTest Procedure (two sided tests). We considered a $\mathrm{P}$ value of $<0.05$ to be significant.

\section{Results}

Of 1800 letters mailed, 1598 members were both reachable by mail and potentially eligible to participate. Of these, 853 completed the telephone interview, 312 refused to participate, 419 were not reachable by telephone after five attempts, and 14 were not interviewed because the target number had been reached. The response rate was $53.4 \%$ of all people who were potentially eligible to participate (853/1598) and $73.2 \%$ $(853 / 1165)$ of all who could be reached by phone.

Non-respondents did not differ significantly from respondents, except for a somewhat greater proportion of women among the respondents.

\section{Respondent characteristics}

Respondents were primarily women $(n=524,61.4 \%)$, with an average age of 45 years. At least an undergraduate college education was attained by $43.6 \%(n=372)$. Although all respondents had been seen by a doctor for one or more chronic conditions, most reported being in excellent $(n=170,19.9 \%)$ or very good $(\mathrm{n}=359,42.1 \%)$ health (table $1 \Downarrow)$.

\section{Mind-body influences on health}

Most respondents believed that a person's mind can affect their health $(96.4 \%, 95 \%$ confidence interval $95.1 \%$ to $97.6 \%)$, that thinking positively can improve the physical symptoms of an illness $(9.51 \%, 89.6 \%$ to $93.4 \%)$, and that a doctor speaking positively about a medicine or medical treatment can have at least a fair amount of influence on the patient's response to that treatment $(85.0 \%, 82.6 \%$ to $87.4 \%)$. 


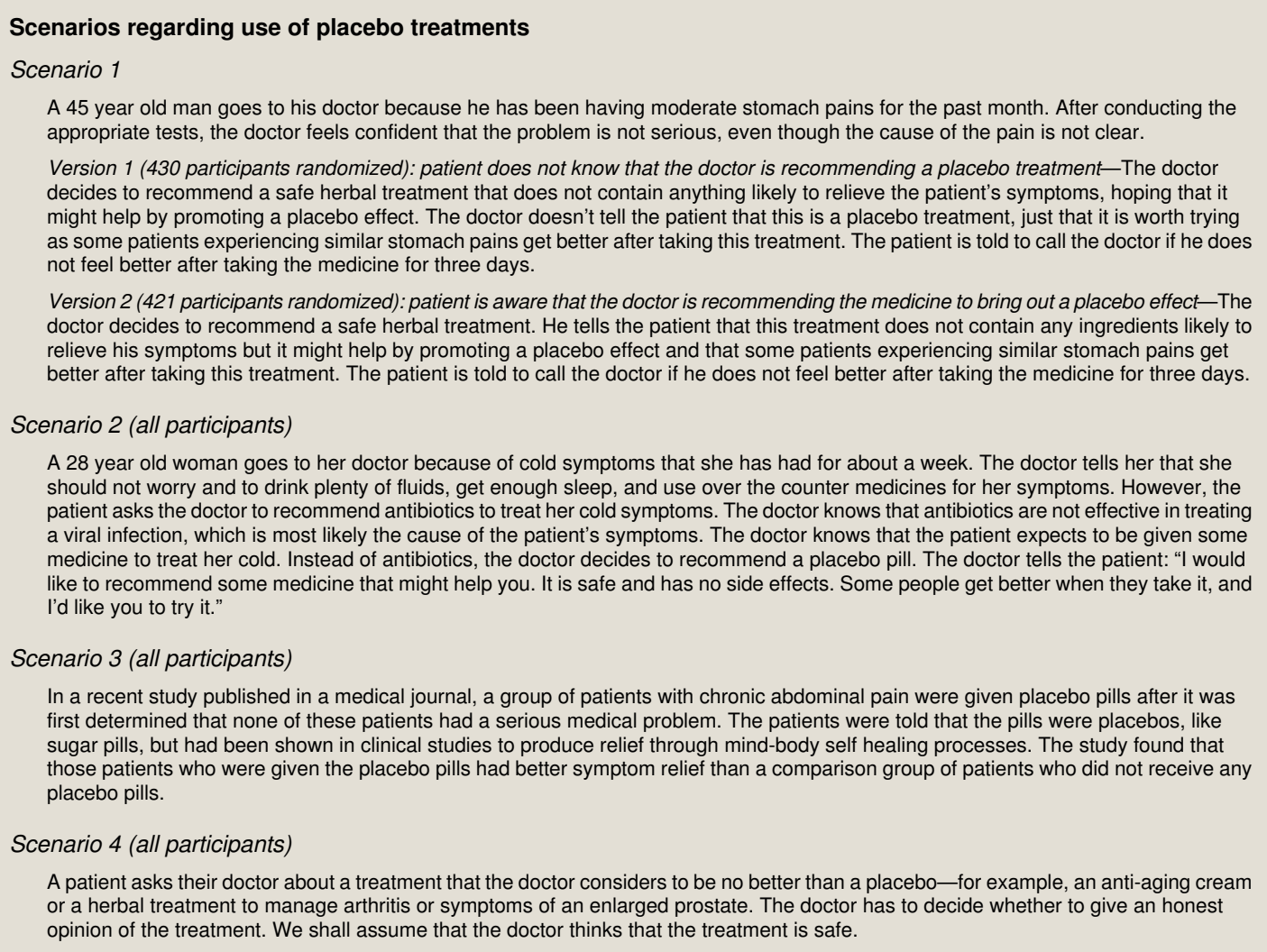

\section{Familiarity with placebo terminology and how placebo treatments work}

While $80.7 \%$ ( $78.0 \%$ to $83.3 \%$ ) of respondents were familiar with the term "placebo" prior to being provided the definition, only $51.5 \%$ ( $48.1 \%$ to $54.8 \%$ ) had heard the term "placebo effect." Approximately two thirds (69.4\%, 66.3\% to 72.5\%) of respondents believed that placebo treatments can produce physical changes in the body (table $2 \Downarrow$ ). Respondents were asked several questions about the extent to which they believe placebos are effective when patients either do or do not know they are receiving a placebo treatment. Over $80 \%(83.1 \%, 80.5 \%$ to $85.7 \%$ ) of respondents believed that placebo treatments can only be effective if patients do not know that they are receiving a placebo treatment and trust the physician who is recommending the treatment $(81.0 \%, 78.3 \%$ to $83.6 \%)$ (table 2). However, in response to scenario 3 reflecting the randomized trial of open placebo in patients with irritable bowel syndrome (box),${ }^{14} 63.9 \%$ (59.3\% to $68.6 \%$ ) thought that a placebo treatment might work to relieve a patient's stomach pain even if the patient knew it was a placebo.

\section{General acceptability of prescribing placebo treatments}

Only $21.9 \%$ (19.1\% to $24.7 \%$ ) of respondents judged that it was never acceptable for doctors to recommend placebo treatments (table 2). While $76.2 \%$ ( $73.4 \%$ to $79.1 \%$ ) judged it was acceptable for doctors to recommend a placebo treatment if they thought it would benefit and not harm the patient, fewer $(50.1 \%$, $46.7 \%$ to $53.4 \%$ ) considered it acceptable if doctors were uncertain of the benefit. Approximately $70.0 \%$ (66.9\% to $73.1 \%$ ) stated it was acceptable for doctors to offer a safe placebo treatment if it addressed the need of patients to feel like they were being given something to get better. Respondents were divided in their views about whether doctors should call a placebo treatment "real medicine"; $46.4 \%$ (43.0\% to $49.8 \%$ ) agreed and $53.6 \%(50.2 \%$ to $57.0 \%)$ disagreed that it is acceptable for physicians to do so (table 2).

Approximately $74.7 \%$ (71.8\% to $77.6 \%$ ) of respondents thought it would be acceptable for a physician to offer a safe placebo treatment for moderate stomach pain of unknown cause that the doctor believed was not serious (both versions of scenario 1 ; table $3 \Downarrow$ ), and $65.6 \%$ (62.3\% to $68.8 \%)$ thought it would be acceptable to offer a safe placebo treatment for someone who instead had wanted a prescription for antibiotics for cold symptoms (scenario 2; table 3). In that scenario, 92.1\% (90.3\% to $93.9 \%$ ) judged it not appropriate for doctors to prescribe antibiotics. In the open placebo scenario (scenario 3; table 3), $84.9 \%(82.5 \%$ to $87.3 \%)$ of respondents thought that it was acceptable for patients to be given placebo treatments to treat chronic abdominal pain.

\section{Placebo treatments, honesty, and trust}

Several responses emphasize the value of physician honesty. Specifically, in response to the first version of scenario 1 in which physicians offer patients a placebo treatment for stomach pain without indicating that it is a placebo treatment (box), $74.5 \%(70.4 \%$ to $78.7 \%)$ of respondents agreed that if the treatment worked and patients asked what the medicine was, the doctors should tell them it was a placebo. In the cold symptom scenario (scenario 2), respondents were asked how the patient's future relationship with her doctor would be affected if she later found out that she had been given a placebo treatment. Nearly twice as many respondents thought that discovering that she had received the placebo treatment, even if it worked, would have a negative rather than positive effect $(53.9 \%(50.5 \%$ to $57.3 \%) v 28.5 \%(25.4 \%$ to $31.6 \%))$ on the future doctor-patient relationship; only $17.6 \%$ (15.0\% to $20.2 \%$ ) thought that use of the placebo treatment would have no effect on their future relationship. If the placebo treatment didn't work, 
$81.2 \%$ ( $78.5 \%$ to $83.8 \%$ ) of respondents judged that this would have a negative effect on the doctor-patient relationship.

Respondents were read scenario 4 (box) asking whether doctors should give patients an honest opinion about a treatment the doctors considered safe but no better than a placebo. When the patient in the scenario had only read about the treatment but not yet tried it, $63.3 \%$ (60.1\% to $66.6 \%)$ of respondents definitely thought doctors should give an honest assessment of the treatment (table $4 \Downarrow$ ), $49.6 \%$ (46.2\% to $53.0 \%$ ) when the treatment had been recommended by a friend who thought it had helped, and just $33.9 \%$ (30.7\% to $37.1 \%$ ) when patients reported that they had been using the treatment and it seemed to be helping. Moreover, if patients felt that they were receiving benefit from using the treatment, $18.2 \%$ (15.7\% to $20.8 \%$ ) of respondents thought that doctors should definitely not tell patients that the treatment was no better than a placebo.

\section{Willingness to try placebo treatments}

Respondents randomized to the second version of scenario 1 (box) were asked if they would be willing to take a placebo treatment for moderate stomach pain if they were aware that the doctor was recommending a placebo treatment. Two thirds of respondents $(65.4 \%, 60.8 \%$ to $70.0 \%)$ said they would probably $(36.5 \%, 31.9 \%$ to $41.1 \%)$ or definitely $(28.9 \%, 24.5 \%$ to $33.2 \%$ ) be willing to take the treatment under these conditions.

Similarly, in response to scenario 3 (box), over half of respondents $(61.5 \%, 58.2 \%$ to $64.8 \%)$ said they would probably $(30.9 \%, 27.8 \%$ to $34.0 \%)$ or definitely $(30.6 \%, 27.5 \%$ to $33.7 \%)$ take pills for chronic abdominal pain if they knew the pills were placebo treatments intended to produce relief through mind-body self healing processes.

\section{Discussion}

The opinions of US patients have been missing from debates over the use of placebo treatments in clinical practice and deliberate efforts by physicians to enhance patient care by promoting placebo responses. Our data show that patients are open to the idea of placebo treatments. Most (50-84\%) judged it acceptable for doctors to recommend placebo treatments under conditions that varied according to the doctor's level of certainty about the benefits of the treatment, the purpose of the treatment (for example, to address a patient's need to receive a treatment), and the transparency with which the treatment was described to patients. Fewer than a quarter stated that it was never acceptable for doctors to recommend placebo treatments. In addition, many respondents indicated a willingness to try placebo treatments in different scenarios. This is generally compatible with trends reported in previous patient surveys in other countries regarding willingness to try placebo treatments. ${ }^{16-18} 20$

Our findings also underscore the importance of honesty and trust in the prescription of placebo treatments. Respondents indicated that the use of placebo treatments could have a negative impact on the doctor-patient relationship if patients learnt that a doctor had recommended a placebo to placate patient's expectations for treatment-especially if it did not work. Respondents said that doctors should be honest about an intervention being a placebo treatment when patients ask specific questions about the treatment, and they disagreed about whether it is acceptable for physicians to call a placebo treatment "real medicine." Interestingly, some respondents thought that doctors should not disclose that a treatment is a placebo if it is working, suggesting some support for the non-transparent use of placebo treatments, a finding not reported in previous surveys.
Although clinical practice guidelines recommend against the use of placebo treatments to mollify patients, ${ }^{10}$ most participants in this survey judged their use to be acceptable to address patients' need to feel like they received some treatment. Patients did, however, specify limits on how far physicians should go to placate patients, with over $90 \%$ judging it inappropriate for doctors to prescribe antibiotics for a cold, even when patients ask for it. Yet recent surveys suggest that a small proportion of physicians would indeed prescribe antibiotics to some patients in this situation, and that they had done so at least once within the past year. ${ }^{41}$ Taken together, these findings hint at a disconnect between clinical practice guidelines, patients' opinions, and physicians' practices that should be further explored.

Although our data indicate a general acceptance of placebo treatments by patients, responses to different questions about the effectiveness of such treatments varied. While most thought when asked directly that placebo treatments are only effective when patients do not know they are receiving them, the responses to the more nuanced scenarios indicated a general acceptance of transparently prescribed "open" placebo treatments - both a belief that they are effective (62\%) and a willingness to take them (61-65\%). This discrepancy prompts questions about how well patients understand the concept of placebo treatments in general and whether providing additional detail in the scenarios adds to their understanding such that their answers to the corresponding questions are more accurate.

The study sample was representative of the population of Northern California but may not be representative of US patients in general. Our sample was more highly educated $(\geq 44 \%$ college graduates) than the general population, had health insurance coverage through Kaiser Permanente Northern California, and had seen a physician within the past six months for a chronic medical condition. Although we constructed our definition of placebo treatments based on feedback from focus groups and pretest interviews, respondents may none the less have variable or limited understandings of the concepts of the placebo effect and placebo treatments. This is perhaps not surprising, given the variable understandings of these concepts among the placebo research community. ${ }^{5}$ In view of the widespread variability in definitions and conceptions of placebo in the literature, we endeavored to employ a pragmatic definition of the placebo effect in our questionnaire that would be useful in the context of a patient survey. While there may be some vagueness and ambiguity in the concept of placebo treatments, the pattern of survey responses and our experience in exploring placebo treatments with focus groups indicate that patients are able to understand the difference between treatments that work on the basis of their inherent pharmacologic properties and those that may produce benefit primarily by means of positive expectations. We observed some variability in answers to analogous questions that were asked in different ways (for example, directly versus in contextualized scenarios), and it is difficult to know which formulation of the questions is likely to reflect attitudes more accurately. Furthermore, the survey captures patients' opinions about a series of plausible hypothetical scenarios rather than actual behaviors and experiences.

Researchers of one study have argued that models of shared decision making need to include conversations between physicians and patients about the role of the placebo effect in clinical care, ${ }^{22}$ and clinical practice guidelines leave the door open for the use of placebo treatments when presented to patients transparently..$^{10}$ Researchers of another study further suggest that attitudinal data can help develop and foster the use of 
effective and non-deceptive placebo treatment techniques. ${ }^{20}$ That many patients in this survey seem favorable to the idea of placebo treatments suggests that physicians should consider engaging with patients to discuss their values and attitudes concerning the appropriateness of using placebos in the context of clinical decision making. Such conversations could allow physicians to determine which patients might be open to the use of placebo treatments and could help physicians tailor their description of placebo treatments according to patients' preferences and level of understanding. Further research is needed to determine how physicians can optimally promote placebo responses in clinical practice and to guide the appropriate use of placebo treatments.

We thank Christine Grady and Justin Lowenthal for their helpful review of emerging drafts of this manuscript.

Contributors: $\mathrm{SCH}$ designed the survey instrument, monitored data collection for the survey, analyzed data, and drafted and revised the paper. She is guarantor. LC designed the survey instrument, analyzed data, and revised the paper. AA, NPG, and CPS designed the survey instrument, implemented the survey within Kaiser Permanente Northern California, monitored data collection for the survey, developed the statistical analysis plan, cleaned and analysed the data, and revised the paper. TPK designed the survey instrument and revised the paper. FGM initiated the collaborative project, designed the survey instrument, monitored data collection for the survey, analyzed data, and revised the paper. All authors, external and internal, had full access to all of the data (including statistical reports and tables) in the study and can take responsibility for the integrity of the data and the accuracy of the data analysis.

Funding: This research was supported by the National Center for Complementary and Alternative Medicine, the Clinical Center, and the intramural research program of the National Human Genome Research Institute, National Institutes of Health. The opinions expressed are those of the authors and do not necessarily reflect the policies or views of the National Institutes of Health or the Department of Health and Human Services. The study funders played no role in the study design; collection, analysis, and interpretation of data; writing of the report; or the decision to submit the article for publication. Researchers were independent of influence from study funders.

Competing interests: All authors have completed the ICMJE uniform disclosure form at www.icmje.org/coi_disclosure.pdf (available on request from the corresponding author) and declare: no support from any organisation for the submitted work; no financial relationships with any organisations that might have an interest in the submitted work in the previous three years, and no other relationships or activities that could appear to have influenced the submitted work.
Ethical approval: All procedures were approved by the Kaiser Foundation Research Institute institutional review board, CN-10AAvin-02-H; and classified as exempt from review by the institutional review board at the National Institutes of Health.

Data sharing: No additional data available.

1 Nizan U, Lichenberg P. Questionnaire survey on use of placebo. BMJ 2004;329:944-6.

2 Kermen R, Hickner J, Brody H, Hasham I. Family physicians believe the placebo effect is therapeutic but often use real drugs as placebos. Fam Med 2010;42:636-42.

3 Fassler M, Meissner K, Schneider A, Linde K. Frequency and circumstance of placebo use in clinical practice-a systematic review of empirical studies. BMC Med 2010;8:15-24

4 Tilburt JC, Emanuel EJ, Kaptchuk TJ, Curlin FA, Miller FG. Prescribing 'placebo treatments': results of a national survey of US internists and rheumatologists, BMJ 2008;337:a1938.

5 Louhiala P. What do we really know about the deliberate use of placebos in clinical practice. J Med Ethics 2012;38:403-5.

6 Colloca L, Miller FG. Harnessing the placebo effect: the need for translational research. Phil Trans Soc B 2011;366:1922-30.

7 O'Neill O. Paternalism and partial autonomy. J Med Ethics 1984;10:173-8.

8 Brody H. The lie that heals: the ethics of giving placebos. Ann Intern Med 1982;97:112-8.

9 Miller FG, Colloca L. The legitimacy of placebo treatments in clinical practice: evidence and ethics. Am J Bioeth 2009;9:39-47.

10 American Medical Association. American Medical Association Code of Medical Ethics, Opinion 8.083: Placebo Use in Clinical Practice. 2007. www.ama-assn.org/ama/pub/ physician-resources/medical-ethics/code-medical-ethics/opinion8083.shtml.

11 Boozang KM. The therapeutic placebo: the case for patient deception. Fla Law Rev 2002;54:687-746.

12 Kolber A. A limited defense of clinical placebo deception. Yale Law Policy Rev 2007;26:75-134.

13 Finniss DG, Kaptchuk TJ, Miller FG, Benedetti F. Biological, clinical, and ethical advances of placebo effects. Lancet 2010;375:686-95.

14 Kaptchuk TJ, Friedlander E, Kelley JM, Sanchez MN, Kokkotou E, Singer JP, et al. Placebos without deception: a randomized controlled trial in irritable bowel syndrome. PLoS One 2010;5:e15591

15 Bishop FL, Jacobson EE, Shaw J, Kaptchuk TJ. Scientific tools, fake treatments or triggers for psychological healing: how RCT participants conceptualize placebos. Soc Sci Med 2012;8:1138-49.

16 Chen GF, Johnson MH. Patients' attitudes to the use of placebos: results from a New Zealand survey. N Z Med J 2009;122:35-46.

17 Berthelot JM, Maugars $\mathrm{Y}$, Abgrall M, Prost A. Interindividual variations in beliefs about the placebo effect: a study in 300 rheumatology inpatients and 100 nurses. Joint Bone Spine 2001;68:65-70.

18 Lynoe N, Mattsson B, Sandlund M. The attitudes of patients and physicians towards placebo treatment-a comparative study. Soc Sci Med 1993;36:767-74.

19 Gordon NP. Similarity of the adult Kaiser Permanente membership in Northern California to the insured and general population in Northern California: statistics from the 2009 California Health Interview Survey. Internal Division of Research Report. 2012. Kaiser Permanente Division of Research. www.dor.kaiser.org/external/chis non kp 2009/.

20 Fassler M, Gnadinger M, Rosemann T, Biller-Andorno N. Placebo interventions in practice: a questionnaire survey on the attitudes of patients and physicians. Br J Gen Pract 2011;61:101-7.

21 Hrobjartsson A, Norup M. The use of placebo interventions in medical practice-a national questionnaire survey of Danish clinicians. Eval Health Prof 2003;26:153-65.

22 Brody H, Colloca L, Miller FG. The placebo phenomenon: implications for the ethics of shared decisionmaking. J Gen Intern Med 2012;27:739-42.

\section{Cite this as: BMJ 2013;346:f3757}

This is an Open Access article distributed in accordance with the Creative Commons Attribution Non Commercial (CC BY-NC 3.0) license, which permits others to distribute, remix, adapt, build upon this work non-commercially, and license their derivative works on different terms, provided the original work is properly cited and the use is non-commercial. See: http://creativecommons.org/licenses/by-nc/3.0/. 


\section{What is already known on this topic}

The use of placebo treatments by physicians in clinical practice has been documented in recent surveys Attitudinal surveys in other countries suggest that patients are open to the use of placebo treatments in specific circumstances

\section{What this study adds}

This study provides data on US patients' attitudes about the use of placebo treatments in clinical practice

These data suggest that many patients are favorable to the idea of placebo treatments

\section{Tables}

\section{Table 1/ Demographic data. Values are number (percentage) of participants unless stated otherwise}

\begin{tabular}{|c|c|}
\hline Characteristics & Participants ( $n=853$ ) \\
\hline Women & $524(61.4)$ \\
\hline Men & $329(38.6)$ \\
\hline \multicolumn{2}{|l|}{ Age group (years): } \\
\hline $18-35$ & $280(32.8)$ \\
\hline $36-60$ & $408(47.8)$ \\
\hline$\geq 61$ & $165(19.3)$ \\
\hline Mean (SD) age & $45.1(15.7)$ \\
\hline \multicolumn{2}{|l|}{ Educational level: } \\
\hline Less than high school graduate & $13(1.5)$ \\
\hline High school graduate & $107(117.1)$ \\
\hline Some college & $316(37.4)$ \\
\hline College degree or beyond & $372(44.5)$ \\
\hline \multicolumn{2}{|l|}{ Race/ethnicity: } \\
\hline White non-Hispanic & $486(57.7)$ \\
\hline African-American/other black & $82(9.7)$ \\
\hline Hispanic/Latino & $132(15.7)$ \\
\hline Asian non-Hispanic & $121(14.4)$ \\
\hline American Indian or Alaska Native & $4(0.5)$ \\
\hline Native Hawaiian or other Pacific Islander & $7(0.8)$ \\
\hline Mixed & $10(1.2)$ \\
\hline \multicolumn{2}{|l|}{ Self reported health: } \\
\hline Excellent & $170(20.1)$ \\
\hline Very good & $359(42.4)$ \\
\hline Good & $234(27.6)$ \\
\hline Fair & $69(8.1)$ \\
\hline Poor & $15(1.8)$ \\
\hline
\end{tabular}

*Based on Office of Management and Budget categories (www.whitehouse.gov/sites/default/files/omb/assets/information_and_regulatory_affairs/re_app-a-update. pdf). 


\begin{tabular}{|c|c|c|c|c|}
\hline \multirow[b]{2}{*}{ Statements } & \multicolumn{4}{|c|}{ Opinions ( $\%$ of participants, $95 \% \mathrm{Cl}$ ) } \\
\hline & Strongly agree & Somewhat agree & Somewhat disagree & Strongly disagree \\
\hline $\begin{array}{l}\text { Placebo treatments can produce physical changes } \\
\text { in the body }\end{array}$ & 20.1 (17.4 to 22.8$)$ & 49.4 (46.0 to 52.7$)$ & 19.8 (17.1 to 22.5$)$ & 10.7 (8.7 to 12.8$)$ \\
\hline $\begin{array}{l}\text { For a placebo treatment to be effective, patients } \\
\text { cannot know that they are receiving a placebo } \\
\text { treatment }\end{array}$ & 68.7 (65.6 to 71.9$)$ & $14.3(12.0$ to 16.7$)$ & $7.3(5.6$ to 9.1$)$ & 9.6 (7.6 to $11.6 \mathrm{~S})$ \\
\hline $\begin{array}{l}\text { Placebo treatments can only be effective if patients } \\
\text { trust the physicians who recommend the treatment }\end{array}$ & 50.7 (47.3 to 54.0$)$ & $30.3(27.2$ to 33.4$)$ & $12.4(10.1$ to 14.6$)$ & 6.77 (5.0 to 8.3$)$ \\
\hline $\begin{array}{l}\text { Doctors should never recommend placebo } \\
\text { treatments to patients }\end{array}$ & $11.4(9.2$ to 13.5$)$ & 10.5 (8.5 to 12.6$)$ & 38.7 (35.4 to 42.0$)$ & $39.4(36.1$ to 42.7$)$ \\
\hline $\begin{array}{l}\text { It is acceptable for doctors to recommend a placebo } \\
\text { treatment if they believe the treatment will benefit } \\
\text { the patients and not cause harm }\end{array}$ & $31.9(28.8$ to 35.1$)$ & $44.3(41.0$ to 47.7$)$ & $12.4(10.2$ to 14.6$)$ & $11.3(9.2$ to 13.5$)$ \\
\hline $\begin{array}{l}\text { It is acceptable for doctors to recommend a placebo } \\
\text { treatment if they are uncertain about whether the } \\
\text { treatment will provide a benefit for patients, so long } \\
\text { as the doctors believes it is safe }\end{array}$ & $14.2(11.8$ to 16.6$)$ & 35.9 (32.6 to 39.1$)$ & 25.6 (22.6 to 28.5$)$ & 24.4 (21.5 to 27.3$)$ \\
\hline $\begin{array}{l}\text { In general, it is acceptable for doctors to offer a } \\
\text { safe placebo treatment if it addresses the patients' } \\
\text { need to feel like they are being given something to } \\
\text { get better }\end{array}$ & $30.8(27.7$ to 34.0$)$ & 39.1 (35.8 to 42.4$)$ & $13.2(10.9$ to 15.5$)$ & $16.8(14.3$ to 19.4$)$ \\
\hline $\begin{array}{l}\text { If doctors recommend a placebo treatment, it is } \\
\text { acceptable for the doctors to tell the patients that } \\
\text { this treatment is a real medicine }\end{array}$ & 20.5 (17.8 to 23.3$)$ & 25.9 (22.9 to 28.8$)$ & $21.0(18.3$ to 23.8$)$ & 32.6 (29.4 to 35.7$)$ \\
\hline \multicolumn{5}{|c|}{$\begin{array}{l}\text { *Placebo treatment was explained: "A patient experiences a placebo effect when they get better after taking a treatment, not because of the treatment itself, but } \\
\text { because the patient expects they will benefit from the treatment. For the rest of this survey, we will ask you to assume that a placebo treatment is a treatment that } \\
\text { only works because it can produce a placebo effect. Placebo treatments can be sugar pills or other treatments used to create a placebo effect." } \\
\text { †Response options for this item were: definitely yes, probably yes, probably not, definitely not. }\end{array}$} \\
\hline
\end{tabular}


Table 3| Acceptability of offering placebo treatments in different scenarios*

\begin{tabular}{lll} 
& & \multicolumn{2}{c}{$\%$ of participants $(95 \% \mathrm{Cl})$} \\
\cline { 3 - 3 } Treatment scenarios & Definitely yes Probably yes Probably not Definitely not
\end{tabular}

Acceptable for doctors to try placebo treatments for stomach pain when patients 30.9 (26.5 to 35.3$) \quad 40.0$ (35.4 to 44.7$) \quad 8.9$ (6.2 to 11.6$) \quad 20.1$ (16.3 to 24.0$)$ do not know doctors are recommending a placebo treatment? (Scenario 1, version

1) $(n=427)$

Acceptable for doctors to try a placebo treatment for stomach pain when patients 41.1 (36.3 to 45.8$) \quad 37.5$ (32.8 to 42.1$) \quad 8.1$ (5.5 to 10.7$) \quad 13.4$ (10.1 to 16.6$)$ are aware that doctors are recommending a placebo treatment? (Scenario 1, version 2) $(\mathrm{n}=419)$

Acceptable for doctors to give patients placebo treatment for cold symptoms if $\quad 36.6$ (33.3 to 39.80$) 29.0$ (25.9 to 32.0$) 9.70$ (7.7 to 11.7$) 24.7$ (21.8 to 27.6 ) patients are told that they are getting medicine that is safe, has no side effects, and helps some people get better when they take it? (Scenario 2) treat cold symptoms that are likely to be caused by a viral infection? (Scenario 3)

Acceptable for doctors to give patients placebo pills for abdominal pain if patients 57.6 (54.2 to 60.9) 27.3 (24.3 to 30.4$) \quad 7.3$ (5.5 to 9.0$)$ are told they are getting placebo medicine to produce relief through mind-body self healing processes? (Scenario 3) 
Table 4| Scenarios for query on whether doctors should tell patients when they think a treatment is safe but no better than a placebo*

Scenarios $\quad$\begin{tabular}{c}
$\%$ of participants $(95 \% \mathrm{Cl})$ \\
\cline { 3 - 4 } Probably not Definitely not
\end{tabular}

If patients tell the doctor that they read about the treatment on the internet or in a 63.3 (60.1 to 66.6$) 22.7$ (19.9 to 25.6$) \quad 8.3$ (6.5 to 10.2$) \quad 5.6$ (4.0 to 7.2$)$ magazine but have not tried it yet

If patients tell the doctor that a good friend is using the treatment and felt it was $\quad 49.6$ (46.2 to 53.0$) 27.7$ (24.7 to 30.7$) \quad 14.6$ (12.2 to 17.0$) \quad 8.1$ (6.2 to 9.90$)$ really helping

If patients tell the doctor that they have been using the treatment for the past two 33.9 (30.7 to 37.1$) 22.5$ (19.7 to 25.3$) 25.4$ (22.4 to 28.3 ) 18.2 (15.6 to 20.8 ) weeks and it seems to be helping 